\title{
Existence results for a non-resonant nonlocal boundary value problems
}

\author{
Katarzyna Szymaìska-Dębowska
}




\section{EXISTENCE RESULTS FOR A NON-RESONANT NONLOCAL BOUNDARY VALUE PROBLEMS}

\section{KATARZYNA SZYMAŃSKA-DȨBOWSKA}

Received 24 September, 2013

Abstract. In this paper we consider the following nonlocal boundary value problems

$$
\left(p(t) x^{\prime}\right)^{\prime}=f\left(t, x, x^{\prime}\right), \quad x^{\prime}(0)=0, \quad x(1)=\int_{0}^{1} x^{\prime}(s) d g(s)
$$

and

$$
\left(p(t) x^{\prime}\right)^{\prime}=f\left(t, x, x^{\prime}\right), \quad x^{\prime}(0)=0, \quad x(1)=\int_{0}^{1} x(s) d g(s),
$$

where $f:[0,1] \times \mathbb{R}^{\mathrm{k}} \times \mathbb{R}^{\mathrm{k}} \rightarrow \mathbb{R}^{\mathrm{k}}$ and the integrals are meant in the sense of Riemann-Stieltjes. Under a sign condition on the function $f$, we prove the existence of solutions.

2010 Mathematics Subject Classification: 34B10; 34B15

Keywords: nonlocal boundary value problem, non-resonant boundary value problem, LeraySchauder degree theory

\section{INTRODUCTION}

In this paper the following ordinary differential equations

$$
\left(p(t) x^{\prime}\right)^{\prime}=f\left(t, x, x^{\prime}\right), \quad t \in[0,1]
$$

with the initial condition

$$
x^{\prime}(0)=0
$$

and the non-local boundary condition

$$
x(1)=\int_{0}^{1} x^{\prime}(s) d g(s)
$$

or

$$
x(1)=\int_{0}^{1} x(s) d g(s)
$$


where $f=\left(f_{1}, \ldots, f_{k}\right):[0,1] \times \mathbb{R}^{\mathrm{k}} \times \mathbb{R}^{\mathrm{k}} \rightarrow \mathbb{R}^{\mathrm{k}}$ is continuous, $p$ is of class $C^{1}$, $p(t)>0$ and $p^{\prime}(t) \geq 0, t \in[0,1], g=\left(g_{1}, \ldots, g_{k}\right):[0,1] \rightarrow \mathbb{R}^{\mathrm{k}}$ has bounded variation and $\int_{0}^{1} x^{\prime}(s) d g(s)=\left[\int_{0}^{1} x_{1}^{\prime}(s) d g_{1}(s), \ldots, \int_{0}^{1} x_{k}^{\prime}(s) d g_{k}(s)\right], \int_{0}^{1} x(s) d g(s)=$ $\left[\int_{0}^{1} x_{1}(s) d g_{1}(s), \ldots, \int_{0}^{1} x_{k}(s) d g_{k}(s)\right]$, is studied.

In other words, for instance, (1.1), (1.2) and (1.3) is the system of $k$ BVPs

$$
\left\{\begin{array}{l}
\left(p(t) x_{i}^{\prime}(t)\right)^{\prime}=f_{i}\left(t, x(t), x^{\prime}(t)\right) \\
x_{i}^{\prime}(0)=0 \\
x_{i}(1)=\int_{0}^{1} x_{i}^{\prime}(s) d g_{i}(s)
\end{array}\right.
$$

where $t \in[0,1], i=1, \ldots, k$ and the integrals $\int_{0}^{1} x_{i}^{\prime}(s) d g_{i}(s)$ are meant in the sense of Riemann-Stieltjes.

Nonlocal boundary value problems (BVPs) arise in different areas of applied mathematics and physics. Such problems, inter alia, have applications in chemical engineering, thermo-elasticity, underground water flow and population dynamics (see for instance $[1,19]$ and the references therein).

The study of nonlocal BVPs in the case of linear second-order ordinary differential equations was initiated by Bitsadze and Samarski [2] and later continued by Il'in and Moiseev [7, 8]. Since then, many authors began to consider second-order differential equations with various multi-point boundary conditions (compare for instance $[3,5$, 13]).

BVPs with Riemann-Stieltjes integral boundary conditions include as special cases multi-point BVPs. According to the best of our knowledge, [15] is one of the first article in which the Authors consider the Riemann-Stieltjes integral boundary conditions for ordinary differential equations. Nowadays, the problem of the existence of solutions for various types of such nonlocal BVPs is the subject of many papers (we refer the reader to $[10-12,14,18]$ and the references therein).

BVPs of the type: $x^{\prime \prime}=f, x^{\prime}(0)=0$, wherein in the second boundary condition $x$ (1) depends on the derivative of the function $x$ at some of its points, were considered for instance in $[4,6]$. As far as we are aware, the BVP (1.1), (1.2), (1.3) has not been studied in this generality so far.

Nonlocal BVPs with conditions of the type $x^{\prime}(0)=0, x(1)=\int_{0}^{1} x(s) d g(s)$ one can find for instance in [5], [18] or [17]. To our best knowledge, problem (1.1), (1.2), (1.4) was considered for the first time in [9] and [16] - in [9] as a multi-point boundary value problem, in [16] as a nonlocal boundary value problem with the RiemannStieltjes boundary condition.

In all papers mentioned above the Authors deal with scalar problems.

The purpose of this paper is to obtain conditions for the existence of a solution for the BVPs (1.1), (1.2), (1.3) and (1.1), (1.2), (1.4), using the properties of the Leray-Schauder topological degree. Our results extend results in the references. 


\section{MAIN RESUlT}

First, we shall introduce notation and terminology. Denote by $C^{1}\left([0,1], \mathbb{R}^{\mathrm{k}}\right)$ the Banach space of all continuous functions $x:[0,1] \rightarrow \mathbb{R}^{\mathrm{k}}$ which have continuous first derivatives $x^{\prime}$ with the norm

$$
\|x\|=\max \left\{|x(1)|, \sup _{t \in[0,1]}\left|x^{\prime}(t)\right|\right\},
$$

where $|\cdot|$ means the Euclidean norm in $\mathbb{R}^{\mathrm{k}}$.

We shall also need the following result (which is a straight consequence of the classical Arzelà-Ascoli theorem):

Lemma 1. For a set $Z \subset C^{1}\left([0,1], \mathbb{R}^{\mathrm{k}}\right)$ to be relatively compact, it is necessary and sufficient that:

(1) there exists $M>0$ such that for any $x \in Z$ and $t \in[0,1]$ we have $|x(1)| \leq M$ and $\left|x^{\prime}(t)\right| \leq M$;

(2) for every $t_{0} \in[0,1]$ the family $Z^{\prime}:=\left\{x^{\prime} \mid x \in Z\right\}$ is equicontinuous at $t_{0}$.

First let us consider BVP (1.1), (1.2), (1.3). Since the problem is always non-resonant (the homogeneous linear problem has only trivial solution), we will find an equivalent integral equation.

Let us consider the equation (1.1) and integrate it from 0 to $t$, we get

$$
x^{\prime}(t)=\frac{1}{p(t)} \int_{0}^{t} f\left(s, x(s), x^{\prime}(s)\right) d s+\frac{x^{\prime}(0)}{p(t)} .
$$

It then follows from (1.2) that

$$
x^{\prime}(t)=\frac{1}{p(t)} \int_{0}^{t} f\left(s, x(s), x^{\prime}(s)\right) d s .
$$

Now, integrating (2.2) from $t$ to 1 , we have

$$
x(t)=-\int_{t}^{1} \frac{1}{p(s)} \int_{0}^{s} f\left(u, x(u), x^{\prime}(u)\right) d u d s+x(1) .
$$

By (1.3) and (2.3), we obtain

$$
x(1)=\int_{0}^{1} \frac{1}{p(s)} \int_{0}^{s} f\left(u, x(u), x^{\prime}(u)\right) d u d g(s) .
$$

As an immediate corollary we have the following:

Lemma 2. A function $x \in C^{1}\left([0,1], \mathbb{R}^{\mathrm{k}}\right)$ is a solution of the problem (1.1), (1.2), (1.3) only and only if $x$ satisfies the following integral equation

$$
x(t)=-\int_{t}^{1} \frac{1}{p(s)} \int_{0}^{s} f\left(u, x(u), x^{\prime}(u)\right) d u d s+
$$




$$
+\int_{0}^{1} \frac{1}{p(s)} \int_{0}^{s} f\left(u, x(u), x^{\prime}(u)\right) d u d g(s) .
$$

Let $A: \mathrm{C}^{1}\left([0,1], \mathbb{R}^{\mathrm{k}}\right) \rightarrow \mathrm{C}^{1}\left([0,1], \mathbb{R}^{\mathrm{k}}\right)$ be such that

$$
\begin{aligned}
(A x)(t) & =-\int_{t}^{1} \frac{1}{p(s)} \int_{0}^{s} f\left(u, x(u), x^{\prime}(u)\right) d u d s+ \\
& +\int_{0}^{1} \frac{1}{p(s)} \int_{0}^{s} f\left(u, x(u), x^{\prime}(u)\right) d u d g(s) .
\end{aligned}
$$

We thus conclude that

$$
(A x)^{\prime}(t)=\frac{1}{p(t)} \int_{0}^{t} f\left(s, x(s), x^{\prime}(s)\right) d s .
$$

Functions $A x,(A x)^{\prime}:[0,1] \rightarrow \mathbb{R}^{\mathrm{k}}$ are continuous. It follows that the operator $A$ is well-defined.

Let $x \in \mathrm{C}^{1}\left([0,1], \mathbb{R}^{\mathrm{k}}\right)$ and $\|x\|=M$. It is to be noted that

$$
|x(t)| \leq(1-t) \sup _{t \in[0,1]}\left|x^{\prime}(t)\right|+|x(1)| \leq 2 M .
$$

Now, since $f$ is continuous $g$ has bounded variation, by (2.4) and Lemma 1, it is clear that

Lemma 3. The operator $A$ is completely continuous.

The existence result is given in the following theorem:

Theorem 1. Assume that the following conditions hold

(i) $f=\left(f_{1}, \ldots, f_{k}\right):[0,1] \times \mathbb{R}^{\mathrm{k}} \times \mathbb{R}^{\mathrm{k}} \rightarrow \mathbb{R}^{\mathrm{k}}$ is a continuous function;

(ii) there exists $M>0$ such that $(y \mid f(t, x, y))<0$ for $t \in[0,1], x \in \mathbb{R}^{\mathrm{k}}$ and $|y| \geq M$, where $(\cdot \mid \cdot)$ means the scalar product in $\mathbb{R}^{\mathrm{k}}$ corresponding to the Euclidean norm;

(iii) $p \in C^{1}([0,1], \mathbb{R})$ are such that $p(t)>0$ and $p^{\prime}(t) \geq 0$;

(iv) $g=\left(g_{1}, \ldots, g_{k}\right):[0,1] \rightarrow \mathbb{R}^{\mathrm{k}}$ and $\operatorname{Var}(\mathrm{g})<1$, where $\operatorname{Var}(\mathrm{g})$ means the variation of $g$ on the interval $[0,1]$;

Then BVP (1.1), (1.2) and (1.3) has at least one solution.

Proof. Let us consider the continuous family of BVPs:

$$
\left(p(t) \varphi^{\prime}\right)^{\prime}=\lambda f\left(t, \varphi, \varphi^{\prime}\right), \quad \varphi^{\prime}(0)=0, \quad \varphi(1)=\int_{0}^{1} \varphi^{\prime}(s) d g(s),
$$

depending on a parameter $\lambda \in[0,1]$. Notice that the problem (2.5) is equivalent to the following integral equation $\varphi(t)=\lambda A \varphi(t)$. It follows from Lemma 3 that the operator $\lambda A$ is completely continuous. 
Consider the homotopy $H:[0,1] \times \mathrm{C}^{1}\left([0,1], \mathbb{R}^{\mathrm{k}}\right) \rightarrow \mathrm{C}^{1}\left([0,1], \mathbb{R}^{\mathrm{k}}\right)$ given by

$$
H(\lambda, \varphi)=\varphi-\lambda A \varphi
$$

in $\Omega=B(0, M)$, where $M$ is the positive constant from the assumption ( $i i)$.

We shall show that $H(\lambda, \varphi)=0$ has no solution for $\lambda \in[0,1]$ and $\varphi$ belonging to $\partial \Omega$.

Conversely, suppose that $H(\lambda, \varphi)=0$ for $\lambda \in[0,1]$ and $\varphi \in \partial \Omega$.

If $H(\lambda, \varphi)=0$ for $\lambda=0$ and $\varphi \in \partial \Omega$, then BVP (2.5) has only a trivial solution, which contradicts the fact that $\varphi \in \partial \Omega$.

Now, let $H(\lambda, \varphi)=0$ with $\lambda \in(0,1]$ and $\varphi \in \partial \Omega$. By (2.1),

$$
M=\|\varphi\|=\max \left\{|\varphi(1)|, \sup _{t \in[0,1]}\left|\varphi^{\prime}(t)\right|\right\}
$$

First, assume that $|\varphi(1)|=M$. In this case, by (1.3) and (iv), we get

$$
M=|\varphi(1)|=\left|\int_{0}^{1} \varphi^{\prime}(s) d g(s)\right| \leq M \operatorname{Var}(\mathrm{g})<M,
$$

a contradiction.

Now, consider the case when $\sup _{t \in[0,1]}\left|\varphi^{\prime}(t)\right|=M$. Let $\psi(t)=\left|\varphi^{\prime}(t)\right|^{2}$. Notice that $\varphi^{\prime}(0)=0$. Hence $\psi$ has a maximum equal to $M^{2}$ for some $t_{0} \in(0,1]$.

If $t_{0} \in(0,1]$, then, by assumptions (ii), (ii i ) and (2.5), since $\left|\varphi^{\prime}\left(t_{0}\right)\right|=M$, we have

$$
\begin{aligned}
0 \leq \psi^{\prime}\left(t_{0}\right) & =2\left(\varphi^{\prime}\left(t_{0}\right) \mid \varphi^{\prime \prime}\left(t_{0}\right)\right) \\
& =2\left(\varphi^{\prime}\left(t_{0}\right) \mid \frac{\lambda f\left(t_{0}, \varphi\left(t_{0}\right), \varphi^{\prime}\left(t_{0}\right)\right)}{p\left(t_{0}\right)}-\frac{p^{\prime}\left(t_{0}\right)}{p\left(t_{0}\right)} \varphi^{\prime}\left(t_{0}\right)\right) \\
& =2 \frac{\lambda}{p\left(t_{0}\right)}\left(\varphi^{\prime}\left(t_{0}\right) \mid f\left(t_{0}, \varphi\left(t_{0}\right), \varphi^{\prime}\left(t_{0}\right)\right)\right)-2 \frac{p^{\prime}\left(t_{0}\right)}{p\left(t_{0}\right)}\left|\varphi^{\prime}\left(t_{0}\right)\right|^{2}<0 .
\end{aligned}
$$

Hence, we get a contradiction.

Hence homotopy $H$ does not vanish on the boundary of $\Omega$ for $\lambda>0$. We thus conclude that $H(\lambda, \varphi) \neq 0$ for $\lambda \in[0,1]$ and $\varphi \in \partial \Omega$.

Now, by the properties of the Leray-Schauder topological degree, we have

$$
\begin{gathered}
\operatorname{deg}_{L S}(I-A, \Omega)=\operatorname{deg}_{L S}(H(1, \cdot), \Omega) \\
=\operatorname{deg}_{L S}(H(0, \cdot), \Omega)=\operatorname{deg}_{L S}(I, \Omega)=1 \neq 0 .
\end{gathered}
$$

Hence $A$ has a fixed point in $\Omega$, i.e. BVP (1.1), (1.2) and (1.3) has a solution in $\Omega$. 
Now, let us consider BVP (1.1), (1.2), (1.4). For this problem we will assume that $\operatorname{Var}(\mathrm{g})<1 / 2$. If so, then $\int_{0}^{1} d g_{i}(s) \neq 1$ and the problem is non-resonant. Proceeding similarly as in the case of the first problem, we get

Lemma 4. A function $x \in C^{1}\left([0,1], \mathbb{R}^{\mathrm{k}}\right)$ is a solution of the problem (1.1), (1.2), (1.4) only, and only if $x$ satisfies the following integral equation

$$
\begin{aligned}
x_{i}(t) & =-\int_{t}^{1} \frac{1}{p(s)} \int_{0}^{s} f_{i}\left(u, x(u), x^{\prime}(u)\right) d u d s+ \\
& +\alpha_{i}\left[\int_{0}^{1} \int_{s}^{1} \frac{1}{p(u)} \int_{0}^{u} f_{i}\left(z, x(z), x^{\prime}(z)\right) d z d u d g_{i}(s)\right],
\end{aligned}
$$

for every $i=1, \ldots, k$, where $\alpha_{i}:=\left(\int_{0}^{1} d g_{i}(s)-1\right)^{-1}$.

Let $B: \mathrm{C}^{1}\left([0,1], \mathbb{R}^{\mathrm{k}}\right) \rightarrow \mathrm{C}^{1}\left([0,1], \mathbb{R}^{\mathrm{k}}\right)$ is given by

$$
\begin{aligned}
(B x)(t) & =-\int_{t}^{1} \frac{1}{p(s)} \int_{0}^{s} f\left(u, x(u), x^{\prime}(u)\right) d u d s+ \\
& +\alpha\left[\int_{0}^{1} \int_{s}^{1} \frac{1}{p(u)} \int_{0}^{u} f\left(z, x(z), x^{\prime}(z)\right) d z d u d g(s)\right] .
\end{aligned}
$$

It is clear that $B x,(B x)^{\prime}:[0,1] \rightarrow \mathbb{R}^{\mathrm{k}}$ are continuous. It follows that $B$ is welldefined. Moreover, since $f$ is continuous $g$ has bounded variation, by (2.4) and Lemma 1 , it is clear that

Lemma 5. The operator $B$ is completely continuous.

Theorem 2. Assume that the following conditions hold

(i) $f=\left(f_{1}, \ldots, f_{k}\right):[0,1] \times \mathbb{R}^{\mathrm{k}} \times \mathbb{R}^{\mathrm{k}} \rightarrow \mathbb{R}^{\mathrm{k}}$ is a continuous function;

(ii) there exists $M>0$ such that $(y \mid f(t, x, y))<0$ for $t \in[0,1], x \in \mathbb{R}^{\mathrm{k}}$ and $|y| \geq M$

(iii) $p \in C^{1}([0,1], \mathbb{R})$ are such that $p(t)>0$ and $p^{\prime}(t) \geq 0$;

(iv) $g=\left(g_{1}, \ldots, g_{k}\right):[0,1] \rightarrow \mathbb{R}^{\mathrm{k}}$ and $\operatorname{Var}(\mathrm{g})<1 / 2$.

Then BVP (1.1), (1.2) and (1.4) has at least one solution.

Proof. Let us consider the continuous family of BVPs:

$$
\left(p(t) \varphi^{\prime}\right)^{\prime}=\lambda f\left(t, \varphi, \varphi^{\prime}\right), \quad \varphi^{\prime}(0)=0, \quad \varphi(1)=\int_{0}^{1} \varphi(s) d g(s),
$$

depending on a parameter $\lambda \in[0,1]$. Notice that the problem (2.6) is equivalent to the following integral equation $\varphi(t)=\lambda B \varphi(t)$. It follows from Lemma 5 that the operator $\lambda B$ is completely continuous.

Consider the homotopy $H:[0,1] \times \mathrm{C}^{1}\left([0,1], \mathbb{R}^{\mathrm{k}}\right) \rightarrow \mathrm{C}^{1}\left([0,1], \mathbb{R}^{\mathrm{k}}\right)$ given by

$$
H(\lambda, \varphi)=\varphi-\lambda B \varphi
$$


in $\Omega=B(0, M)$, where $M$ is the positive constant from the assumption ( $i i)$.

Notice that $H(\lambda, \varphi)=0$ has no solution for $\lambda \in[0,1]$ and $\varphi$ belonging to the boundary of the ball $\Omega$.

Indeed, $H(0, \varphi)=0$ has only a trivial solution, so $\lambda \neq 0$.

Now, let $H(\lambda, \varphi)=0$ with $\lambda \in(0,1]$ and $\varphi \in \partial \Omega$. By (2.1),

$$
M=\|\varphi\|=\max \left\{|\varphi(1)|, \sup _{t \in[0,1]}\left|\varphi^{\prime}(t)\right|\right\}
$$

First, assume that $|\varphi(1)|=M$. In this case, by (1.4) and (iv), we get

$$
M=|\varphi(1)|=\left|\int_{0}^{1} \varphi(s) d g(s)\right| \leq 2 M \operatorname{Var}(\mathrm{g})<M,
$$

a contradiction. 1

The proof of the fact that $\sup _{t \in[0,1]}\left|\varphi^{\prime}(t)\right| \neq M$ is similar to the proof of Theorem

So, $H(\lambda, \varphi)=0$ has no solution for $\lambda \in[0,1]$ and $\varphi \in \partial \Omega$. Hence $A$ has a fixed point in $\Omega$, i.e. BVP (1.1), (1.2) and (1.4) has a solution in $\Omega$.

\section{Application}

Let us consider the following BVP

$$
\left(p(t) x^{\prime}\right)^{\prime}=f\left(t, x, x^{\prime}\right), \quad x^{\prime}(0)=0, \quad x(1)=\int_{0}^{1} x^{\prime}(s) d g(s),
$$

where $f(t, x, y)=\left(f_{1}(t, x, y), f_{2}(t, x, y)\right)$,

$$
\begin{aligned}
f_{1}(t, x, y) & =-(t+1)\left(\exp \left(-x_{1}^{2}\right)+1\right) y_{1}-(t+1)\left(\exp \left(-x_{2}^{2}\right)+1\right) y_{2}, \\
f_{2}(t, x, y) & =(t+1)\left(\exp \left(-x_{2}^{2}\right)+1\right) y_{1}-(t+1)\left(\exp \left(-x_{1}^{2}\right)+1\right) y_{2}+ \\
& -2(t+1)\left(\exp \left(-x_{1}^{2}\right)+1\right)
\end{aligned}
$$

and $p, g$ are arbitrary functions satisfying the conditions (iii) and (iv).

Observe that $f$ satisfies the assumptions $(i)$ and $(i i)$. Indeed, for any $M>2$ and $|y| \geq M, x \in \mathbb{R}^{2}$ and $t \in[0,1]$, we have

$$
\begin{aligned}
(y \mid f(t, x, y)) & =y_{1}\left(-(t+1)\left(\exp \left(-x_{1}^{2}\right)+1\right) y_{1}-(t+1)\left(\exp \left(-x_{2}^{2}\right)+1\right) y_{2}\right)+ \\
& +y_{2}\left((t+1)\left(\exp \left(-x_{2}^{2}\right)+1\right) y_{1}-(t+1)\left(\exp \left(-x_{1}^{2}\right)+1\right) y_{2}\right)+ \\
& -2 y_{2}(t+1)\left(\exp \left(-x_{1}^{2}\right)+1\right)= \\
& =-(t+1)\left(\exp \left(-x_{1}^{2}\right)+1\right)\left(y_{1}^{2}+y_{2}^{2}+2 y_{2}\right)<0 .
\end{aligned}
$$

Indeed, notice that $y_{1}^{2}+y_{2}^{2}+2 y_{2}>0$, if $y_{2} \in(-\infty,-2] \cup[0, \infty)$. If $y_{2} \in(-2,0)$, we obtain

$$
y_{1}^{2}+y_{2}^{2}+y_{2} \geq M^{2}+y_{2}>2+y_{2}>0 .
$$


Hence, there exists at least one nontrivial solution of (3.1) what ends the example and completes the paper.

\section{REFERENCES}

[1] N. A. Asif, P. W. Eloe, and R. A. Khan, "Positive solutions for a system of singular second order nonlocal boundary value problems," J. Korean Math. Soc., vol. 47, no. 5, pp. 985-1000, 2010.

[2] A. V. Bicadze and A. A. Samarski, "Some elementary generalizations of linear elliptic boundary value problems," Dokl. Akad. Nauk SSSR, vol. 185, pp. 739-740, 1969.

[3] A. M. Dovletov and D. M. Dovletov, "A nonlocal boundary value problem of the 'third kind' for the Sturm-Liouville operator," Izv. Akad. Nauk Turkmen. SSR Ser. Fiz.-Tekhn. Khim. Geol. Nauk, no. 5, pp. 3-6, 1991.

[4] L. H. Erbe, S. C. Hu, and H. Wang, "Multiple positive solutions of some boundary value problems," J. Math. Anal. Appl., vol. 184, no. 3, pp. 640-648, 1994.

[5] W. Feng, "On an m-point boundary value problem," Nonlinear Anal., Theory Methods Appl., vol. 30, no. 8, pp. 5369-5374, 1997.

[6] D. Franco and D. O'Regan, "Existence of solutions to second order problems with nonlinear boundary conditions," Discrete Contin. Dyn. Syst., no. suppl., pp. 273-280, 2003.

[7] V. A. Il'in and E. I. Moiseev, "A nonlocal boundary value problem for the Sturm-Liouville operator in a differential and a difference treatment," Dokl. Akad. Nauk SSSR, vol. 291, no. 3, pp. 534-539, 1986.

[8] V. A. Il'in and E. I. Moiseev, "A nonlocal boundary value problem of the second kind for the Sturm-Louville operator," Differentsial'nye Uravneniya, vol. 23, no. 8, pp. 1422-1431, 1471, 1987.

[9] G. Infante and J. R. L. Webb, "Positive solutions of some nonlocal boundary value problems," Abstr. Appl. Anal., no. 18, pp. 1047-1060, 2003.

[10] G. L. Karakostas and P. C. Tsamatos, "Positive solutions for a nonlocal boundary-value problem with increasing response," Electron. J. Differential Equations, pp. No. 73, 8 pp. (electronic), 2000.

[11] A. Lomtatidze, "On a nonlocal boundary value problem for second order linear ordinary differential equations," J. Math. Anal. Appl., vol. 193, no. 3, pp. 889-908, 1995.

[12] A. Lomtatidze and L. Malaguti, "On a nonlocal boundary value problem for second order nonlinear singular differential equations," Georgian Math. J., vol. 7, no. 1, pp. 133-154, 2000.

[13] R. Ma, "Existence theorems for a second order $m$-point boundary value problem," J. Math. Anal. Appl., vol. 211, no. 2, pp. 545-555, 1997.

[14] R. Ma, "Positive solutions for second order functional differential equations," Nonlinear Stud., vol. 8, no. 2, pp. 239-247, 2001.

[15] Y. V. Pokorny, K. P. Lazarev, and T. M. Gareeva, "Nonlocal boundary value problems for ordinary differential equations," Differentsial'nye Uravneniya, vol. 25, no. 8, pp. 1321-1332, 1466-1467, 1989.

[16] J. R. L. Webb and G. Infante, "Positive solutions of nonlocal boundary value problems: a unified approach," J. London Math. Soc. (2), vol. 74, no. 3, pp. 673-693, 2006.

[17] J. R. L. Webb and M. Zima, "Multiple positive solutions of resonant and non-resonant nonlocal boundary value problems," Nonlinear Anal., vol. 71, no. 3-4, pp. 1369-1378, 2009.

[18] J. R. L. Webb and G. Infante, "Positive solutions of nonlocal boundary value problems involving integral conditions," NoDEA Nonlinear Differential Equations Appl., vol. 15, no. 1-2, pp. 45-67, 2008.

[19] X. Zhang, M. Feng, and W. Ge, "Existence result of second-order differential equations with integral boundary conditions at resonance," J. Math. Anal. Appl., vol. 353, no. 1, pp. 311-319, 2009. 
Author's address

\section{Katarzyna Szymańska-Dębowska}

Technical University of Łódź, Institute of Mathematics, ul. Wólczańska 215, 90 -924 Łódź, Poland

E-mail address: katarzyna.szymanska-debowska@p.lodz.pl 